\title{
Descripción del manejo de la información en la primera infancia en dieciséis municipios colombianos*
}

\section{Description of Information Management in Early Childhood in Sixteen Colombian Municipalities}

\section{Descrição do manuseio da informação na primeira infância em dezesseis municípios colombianos}

Fecha de recepción: 03-04-14 Fecha de aceptación: 09-05-14 Disponible en línea: 01-07-14 doi: 10.11144/Javeriana.rgyps13-27.dmip

Cómo citar este artículo:

Rincón-Rodríguez CJ, Rodríguez-Vargas OE, Galera-Gélvez KG. Descripción del manejo de la información en la primera infancia en dieciséis municipios colombianos. Rev. Gerenc. Polít. Salud. 2014; 13(27): 296-318. http://dx.doi.org/10.11144/Javeriana.rgyps13-27.dmip

Carlos Javier Rincón-Rodríguez**

Olga Eugenia Rodríguez-Vargas***

Katia Cecilia Galera-Gélvez****

Artículo de investigación

** Estadístico, magíster en Epidemiología Clínica, docente investigador de la Facultad de Medicina de la Universidad de La Sabana. Autor principal Correo electrónico: carlosrir@unisabana.edu.co

*** Economista, especialista en Evaluación Social de Proyectos, investigadora del proyecto 3. Monitoreo y Evaluación

296 - Programa Inicio Parejo de la Vida. Monitoreo y Evaluación - Programa Inicio Parejo de la Vida. 


\section{Resumen}

Los objetivos de este trabajo fueron identificar los temas de mayor interés, las principales necesidades de información y los aspectos o sugerencias para la construcción de un sistema de información sobre el cuidado de la salud de la población infantil en los municipios. Se realizaron siete talleres en siete municipios, con la participación de 71 representantes de 19 entidades relacionadas con el cuidado de la salud de la población infantil. Se encontró que la falta de articulación, unificación y consolidación de la información ocasiona que la toma de decisiones se realice a partir de resultados desactualizados, incompletos o inexistentes. Por tanto, en los territorios se necesita un espacio donde se presente un panorama completo de la población infantil, articulando las diferentes instituciones y consolidando periódicamente los temas de interés.

Palabras clave: sistemas de información en salud; desarrollo infantil; cuidado del niño; toma de decisiones; gestión de la información; gestión de la información en salud

\section{Abstract}

The objectives of this work were to identify the most important topics, the main information needs and the aspects or suggestions for the construction of an information system regarding health care of child population in the municipalities. We carried out seven workshops in seven municipalities, with the participation of 71 representatives from 19 entities related to the health care of child population. We found that the lack of coordination, unification and consolidation of information causes decision taking to be performed based on outdated, incomplete or non-existent results. So, territories need a space to show the complete picture of the child population, to coordinate the different institutions and periodically consolidate topics of interest.

Keywords: health information systems; child development; child care; decision taking; information management; health information management

\section{Resumo}

Os objetivos deste trabalho foram identificar os temas de maior interesse, as principais necessidades de informação e os aspectos ou sugestões para a construção de um sistema de informação sobre o cuidado da saúde da população infantil nos municípios. Realizaram-se sete oficinas em sete municípios, com a participação de 71 representantes de 19 entidades relacionadas com o cuidado da saúde da população infantil. Encontrou-se que a falta de articulação, unificação e consolidação da informação ocasiona que a tomada de decisões seja realizada a partir de resultados desatualizados, incompletos ou inexistentes. Por tanto, nos territórios é preciso um espaço onde apresentar um panorama completo da população infantil, articulando as diferentes instituições e consolidando periodicamente os temas de interesse.

Palavras-chave: sistemas de informação em saúde; desenvolvimento infantil; cuidado da criança; tomada de decisões; gestão da informação; gestão da informação em saúde 


\section{Introducción}

En las últimas décadas la primera infancia se ha convertido en una prioridad de los gobiernos a nivel mundial. Tanto es así que en la actualidad cuatro de los ocho objetivos ${ }^{1}$ de las Metas de Desarrollo del Milenio están relacionados con esta población (1). Colombia, por su parte, integró los compromisos adquiridos durante la Cumbre del Milenio de las Naciones Unidas para la construcción de la agenda nacional rectora del tema de la primera infancia. Así, el propósito del Plan Nacional para la Niñez y la Adolescencia 2009-2019 es concentrar esfuerzos para que los niños y las niñas tengan las garantías básicas de sus derechos, mejores condiciones de vida y mejores prioridades (2).

De acuerdo con Health Metrics Network (HMN), en el área de la salud pública se tiene como premisa que mejor información en salud significa mejor toma de decisiones, lo cual a su vez conlleva mejorar el estado de salud de la comunidad en general $(3,4)$. De ahí la importancia de la evaluación de la efectividad de las políticas, planes, programas y proyectos en esta área de interés y de la existencia de sistemas de información, locales, nacionales e internacionales que consoliden información (4).

Ejemplos de sistemas de información regionales que permiten conocer el estado de la primera infancia en algunas áreas son: el de la Organización Mundial de la Salud (wHOSIs) (5), el cual registra 66 indicadores relacionados con este grupo poblacional, y el Sistema de Información Sobre la Primera Infancia en América Latina (SIPI), el cual cuenta con 63 indicadores clasificados en

1 Los cuatro objetivos relacionados con la población infantil son: i) erradicar la pobreza extrema y el hambre; ii) reducir la mortalidad infantil; iii) mejorar la salud materna y iv) Combatir el vih/sida, el paludismo y otras enfermedades. cinco tipos de derechos (6). Por su parte, en el ámbito nacional, cabe mencionar el Plan Decenal de Salud Pública 2012-2021, en el cual 18 de los 94 indicadores de monitoreo se enfocan en la población infantil y materna (7); los diagnósticos de infancia y adolescencia municipales y departamentales que se deben presentar a la Procuraduría General de la Nación (8), donde se incluyen 65 indicadores sociales y 14 financieros; el Sistema Único de Información de la Niñez ${ }^{2}$ (suin), con una priorización inicial de 51 indicadores (9) y el Sistema de Información de la Niñez y la Adolescencia (Sinfonía), creado por el Fondo de las Naciones Unidas para la Infancia (Unicef) (10), el cual consolida más de 100 indicadores de diferentes fuentes oficiales. Otros sistemas, cuya población objetivo no es específicamente la población infantil, pero que registran datos fundamentales de esta, son el Sistema de Vigilancia en Salud Pública (Sivigila) (11) y el Sistema Integral de Información de la Protección Social (Sispro) (12). Otras mediciones importantes son las realizadas por el Departamento Administrativo Nacional de Estadística (DANE), que dentro de su recolección, procesamiento y reporte de información incluyen estadística vitales ${ }^{3}$ (13) y el cálculo de proyecciones poblacionales (14).

Sin embargo, y aunque existen varios sistemas de información en el país, al abordar indicadores relacionados con la primera infancia, la disponibilidad de información estadística no garantiza por sí sola que el proceso de

2 A cargo del Instituto Colombiano de Bienestar Familiar (icbf). Este con el apoyo de otras instituciones tiene como propósito hacer seguimiento al cumplimiento progresivo de los derechos de los niños, niñas y adolescentes, valorando las condiciones socioeconómicas, los riesgos y la vulnerabilidad de los hogares, a partir de fuentes de información ya disponibles.

3 Que son fuente de información de indicadores como razón de mortalidad materna, mortalidad infantil, mortalidad en menores de cinco años por enfermedad respiratoria aguda y por enfermedad diarreica aguda. 
la toma de decisiones sea exitoso (3). Así, aunque buena calidad y oportunidad en los datos son el fundamento de cualquier sistema de información, a menudo se quedan en reportes periódicos o en bases de datos que no son suficientemente utilizadas en la planificación estratégica (15). Otro inconveniente que se puede observar es que los datos son generalmente recolectados y presentados en formatos crudos, sin ninguna síntesis o análisis que apoye la planificación de largo plazo y mejore la comprensión de los formuladores de políticas. Inclusive, cuando los datos están disponibles, en su mayoría se encuentran desactualizados, haciendo la evaluación y seguimiento particularmente difícil (4).

En esta medida, la necesidad de mejorar los sistemas de información ha surgido principalmente de las demandas por la presentación de rendiciones de cuentas y asegurar la toma de decisiones basada en la evidencia (4). Por tanto, es objetivo de los sistemas de información, la producción de información de buena calidad, que las partes interesadas puedan utilizar $(3,4)$.

Esta investigación se encuentra enmarcada dentro de la ejecución del programa Cuidado a la salud de los niños y niñas en edad temprana, orientado por una red de generación y apropiación de conocimiento para la acción, dirigida a potenciar su desarrollo humano y social en la región Sabana Centro de Cundinamarca y cinco municipios de Boyacá (IPV), financiado por Colciencias (convocatoria 537 de 2011) y liderado por la Unión Temporal "Alianza por un Inicio Parejo de la Vida", ${ }^{4}$ el cual contempla dentro de sus objetivos el diseño de un sistema de seguimiento y evaluación como herramienta de monitorización del cuidado de la salud para el desarrollo integral

4 Integrada por la Universidad de La Sabana, la Fundación Santa Fe de Bogotá, la Organización para la Excelencia en Salud y la Fundación Corona. de niños y niñas menores de seis años (CSDINN) en dieciséis municipios. ${ }^{5}$

Este artículo presenta los resultados de la indagación que se realizó en los municipios objetivo, como una etapa previa para el diseño de un sistema de seguimiento y evaluación coherente con las necesidades de los territorios y el manejo de su información. Para esto se definieron tres objetivos: 1) identificar los temas de mayor interés por parte de las instituciones participantes, relacionados con el CSDINN, 2) identificar las principales necesidades en información relacionada con el CSDINN y 3 ) indagar qué aspectos deberían considerarse en la construcción de un Sistema de Información sobre el CSDINN.

\section{Métodología}

Estudio descriptivo donde se realizaron siete talleres participantes, entre el 7 de mayo y el 11 de septiembre del 2013, en siete de los dieciséis municipios donde tiene presencia el programa IPV, los cuales fueron seleccionados de acuerdo con la categorización del Departamento Nacional de Planeación (DNP), ${ }^{6}$ buscando obtener un municipio por cada categoría en Boyacá y Cundinamarca. Los municipios seleccionados para realizar los talleres fueron: Chía ( $2^{\text {a }}$ categoría), Cajicá ( $\left.3^{\text {a }}\right)$, Tenjo (4⿳亠丷a $)$ y Tabio (6 $\left.6^{\mathbf{a}}\right)$ en Cundinamarca y Tunja (2 $\left.2^{\mathrm{a}}\right)$, Duitama ( $\left.3^{\mathrm{a}}\right)$ y Tibasosa $\left(6^{\mathrm{a}}\right)$ en Boyacá.

5 Los 16 municipios donde el programa tiene presencia son: Cajicá, Chía, Cogua, Cota, Gachancipá, Nemocón, Sopó, Tabio, Tenjo, Tocancipá y Zipaquirá (en Cundinamarca), y Duitama, Soatá, Sogamoso, Tibasosa y Tunja (en Boyacá).

6 En Colombia, los municipios están clasificados en seis categorías, dependiendo del número de la población y de los ingresos corrientes de libre destinación, según se determina en la Ley 617 de 2000. Para el presente ejercicio se utilizó la información del Departamento Nacional de Planeación relacionada con la categorización de los municipios en el 2012. 
A los talleres se convocó la presencia de diferentes representantes de entidades públicas, privadas y de participación mixta, con el fin de tener un panorama completo en los municipios, en cuanto a las necesidades de información de las entidades que atienden o desarrollan su objeto social con población infantil. A la convocatoria asistieron en total 71 representantes de las siguientes entidades: Alcaldía (33), Comfaboy (1), comisarías (7), Defensoría del Pueblo (1), Empresas Sociales del Estado (ESE) (8), hogares comunitarios (1), Instituto Colombiano de Bienestar Familiar (1), Inspección de Policía (1), Instituto de Cultura y Bellas Artes (1), Instituto de Recreación y Deporte (5), Instituto para el Desarrollo de Estudios Técnicos (1), juntas de administración local (1), juntas de acción comunal (1), Proniño (1), Personería (3), Red Unidos (1), Registraduría (1) y Veeduría de Salud (3).

La coordinación de los talleres fue realizada por dos personas del equipo de investigación: la primera, cuya labor se centró en la explicación y dirección de la actividad, y la segunda, encargada de realizar una observación registrando comentarios o anotaciones acerca de situaciones relevantes que se presentaron a medida que se desarrollaba la actividad.

Cada uno de los talleres consintió de tres partes (en el anexo 1 se incluye el instrumento utilizado), iniciando con una parte introductoria, la cual comprendía la presentación del propósito del programa IPV y su propuesta de investigación, los objetivos del taller, la agenda de actividades y la firma del consentimiento informado. La segunda parte del taller correspondió a una actividad de trabajo individual, cuya duración fue de aproximadamente 35 minutos, donde cada uno de los participantes debía diligenciar un cuestionario de siete preguntas sobre el manejo de información relacionada con el cuidado de la salud de los niños y niñas menores de seis años en el interior de cada entidad que representaba. La parte final del taller, con una duración de aproximadamente cincuenta minutos, fue una actividad grupal donde se conformaban cuatro grupos y a cada uno se le asignaba una pregunta específica para desarrollar y discutir en el interior de cada grupo. Finalmente, por medio de una cartelera, los participantes compartían sus ideas con respecto a la pregunta formulada con el resto de los asistentes al taller.

Con base en la información capturada, se construyeron categorías de análisis de acuerdo con las respuestas obtenidas; posteriormente, se calculó la frecuencia con que los participantes mencionaron la respuesta dentro de cada una las categorías creadas. En el caso de la pregunta 1, de la parte individual, y la pregunta 4, de la parte grupal, las categorías fueron agrupadas por los cuatro dominios del cuidado de la salud definidos por el Programa IPV: prácticas cotidianas para el cuidado de la salud, preparación para la vida, seguridad e integridad corporal y educación inicial.

\section{Resultados}

\section{Temas relacionados con el CSDINN}

$\mathrm{Al}$ indagar sobre los principales temas relacionados con el CSDINN, en el dominio "Prácticas cotidianas para el cuidado de la salud", se identificaron trece categorías, de las cuales se resaltan siete que fueron mencionadas por diez o más participantes. Estas siete categorías fueron:

- Alimentación/estado nutricional: se encontró una marcada preocupación por la situación nutricional de la primera infancia y de la población en general. En particular, se observaron respuestas como buena alimentación, alimenta- 
ción, dieta, alimentación escolar, alimentación balanceada, buenos hábitos alimenticios, seguridad alimentaria y desórdenes alimenticios y aspectos relacionados con el estado nutricional como desnutrición, perfil de nutrición, bajo peso al nacer y vigilancia nutricional.

- Desarrollo: agrupa las respuestas relacionadas con las diferentes áreas del desarrollo de los niños y niñas menores de seis años, donde se mencionó: comunicación y desarrollo físico, psicomotor, cognitivo, motriz, al igual que aspectos como desarrollo afectivo y social.

- Programas de promoción y prevención: se mostró un gran interés por garantizar la seguridad y protección de la población infantil a través de la prevención, donde los participantes mencionaron aspectos que es necesario prevenir como situaciones de riesgo, trabajo infantil, violencia, maltrato infantil y conducta suicida. En esta categoría se recalcó la importancia de la participación de los padres en estos programas. Adicionalmente, estos programas deben incluir la promoción de estilos de vida saludables y se debe lograr realizar una adecuada difusión de información de estos para que la gente pueda participar.

- Corresponsabilidad de la familia: los participantes mencionaron la importancia de atribuirle la responsabilidad a los padres del cuidado de los niños y niñas. De tal manera, fueron mencionados aspectos como la capacitación de los padres en derechos y deberes de los niños y niñas, la relación afectiva entre padres e hijos, el compromiso por parte de los padres por garantizar las necesidades básicas de los niños y niñas y la importancia de pertenecer a una familia.
- $\quad$ Prácticas de cuidado: se incluyeron las actividades cotidianas que se realizan en el hogar que están relacionadas con el cuidado de los niños y niñas. Fueron mencionados aspectos como el autocuidado, el cuidado personal, el cuidado del cuerpo, la capacitación de madres en cuidados de primera infancia, las prácticas o hábitos saludables y las prácticas tradicionales y su comparación con las prácticas científicas.

- Crecimiento: en esta categoría se recalcó el crecimiento como medida fundamental en la monitorización de los niños, mencionando como aspectos más relevantes los controles de crecimiento y desarrollo y la aplicación de medidas antropométricas.

- Vacunación: se resaltó la vacunación y sus coberturas como tema fundamental en el cuidado de la salud de los niños y niñas menores de seis años.

Con relación al dominio "Preparación para la vida” se identificó únicamente la categoría cuidado de la gestante/lactante, donde se expone la relevancia de los cuidados que se deben tener durante el embarazo y la importancia de la lactancia materna en los primeros meses de vida. De tal forma, se agruparon respuestas como nutrición materno-infantil, maternidad segura, cuidado en el embarazo, fomento de la lactancia materna e importancia de los controles prenatales.

En el dominio "Seguridad e integridad corporal" se construyeron en total nueve categorías de análisis. La más referida fue "Seguridad y protección", que agrupa situaciones de agresión, descuido o no cumplimiento de derechos de los niños. Esta categoría incluye respuestas como violencia intrafamiliar, seguridad en el hogar, protección y restitución de derechos, matoneo, 
seguimiento a procesos administrativos y judiciales donde los niños tienen derechos, abandono, maltrato infantil, abuso sexual, trabajo infantil y prevención de accidentes en casa. La segunda categoría con mayor número de menciones fue "Afiliación/Acceso/ Atención al sGsss", en donde se agruparon respuestas que recalcan la importancia de la cobertura del Sistema General de Seguridad en Salud (sGsss), el acceso a los servicios en las instituciones prestadoras de servicios de salud y la atención con alta calidad y de manera oportuna. Otra categoría fue "Redes y comunicación", donde se incorporaron respuestas que resaltan la necesidad del trabajo articulado entre las instituciones o entidades para brindar un adecuado cuidado de los niños y las niñas, al igual que se resalta la importancia de fomentar la comunicación entre estas instituciones y la comunidad. En esta categoría se incluyeron respuestas como apoyo a instituciones que trabajan en primera infancia, definición de la red de apoyo para los niños y niñas y tener más orientación, capacitación y acompañamiento por parte de entidades como la alcaldía, la comisaria y el centro de salud en los temas de violencia intrafamiliar, maltrato, buen trato, apoyo en las jornadas de vacunación, control de crecimiento y desarrollo. Por último, en la categoría "Condiciones sociodemográficas" se incluyeron las características tanto de los padres como de las condiciones de habitabilidad, mencionando aspectos como el desempleo, la extrema pobreza, la clasificación del Sisben y la focalización de los niños y las niñas.

En el dominio "Educación inicial" se presentaron cuatro categorías, donde la de mayor relevancia se denominó "Educación". Esta agrupa temas relacionados con la identificación de las instituciones educativas y la importancia de facilitar el acceso y alcanzar una alta cobertura. La segunda categoría más referenciada fue "Cultura, recreación y deporte", donde se mencionan temas como ocupación del tiempo libre, jornadas lúdicas e identificación temprana del talento artístico.

Finalmente, se presentaron cuatro categorías adicionales que se pueden interpretar como transversales a los dominios planteados por el programa IPV. Dos de estas categorías con mayor número de menciones fueron: "Estrategias y programas", que integró respuestas donde los asistentes hicieron mención de los diferentes programas y estrategias presentes en los territorios: Desayunos Infantiles, De Cero a Siempre, Hogares Infantiles, Creciendo a Pasitos, Estrategia IAMI, AIEPI, Red Unidos y Programa Nutrir, y "Entornos de política", que incluyó temas como la importancia de fortalecer la política pública, brindar apoyo a los programas que la administración del municipio presenta al concejo, la importancia de articular los proyectos en población de cero a cinco años y la educación formal desde los cinco años y en adelante.

\section{Principales fuentes de información}

Se construyeron cincuenta categorías de análisis que agruparon las principales fuentes de información consultadas regularmente en los municipios. Entre las categorías que fueron mencionadas por once o más participantes están:

- Publicaciones/informes/documentos técnicos: se incluyeron documentos específicos como los planes de desarrollo municipal y departamental, los diagnósticos de infancia y adolescencia municipales y los planes territoriales de salud. De igual forma, se mencionaron diversas publicaciones y documentos técnicos, de manera muy general, como cartillas, folletos, estadísticas, informes institucionales, boletines epidemiológicos departamentales, informes de las Entidades Promotoras de Salud (EPS) 
y de las Instituciones Prestadoras de Servicios de Salud (IPS) e informes de la gobernación.

- Estrategias/programas: los participantes reconocieron como fuentes de información las diferentes estrategias y programas que actúan en el municipio como, por ejemplo: Desayunos Infantiles, IAMI y AIEPI, Red Unidos y Red del Buen Trato. También fueron identificados como fuentes de información diversos proyectos o programas que se realizan en el municipio, entre los que se mencionan el programa de apoyo terapéutico, programas de nutrición, proyectos educativos y programas de formación (arte y lectura para la primera infancia).

- ESE/IPS: las ESE y las IPS fueron identificadas por trece diferentes participantes como importantes fuentes de información, por ser generadores de información primaria básica para la creación de política en salud.

- Sisben: el Sistema de Identificación de Beneficiarios de Potenciales Programas Sociales fue identificado como una importante fuentes de información por doce de los participantes.

- Normas/leyes/lineamiento/resoluciones: los participantes identificaron como fuente de información normatividad relacionada con la población infantil como el Código de Infancia y Adolescencia (Ley 1098), la Resolución 412 sobre los programas de detección temprana y protección específica, la Ley 115 o Ley General de Educación, la Ley General del Deporte (Ley 181) y la Resolución 2121 sobre Patrones de Crecimiento publicados por la Organización Mundial de la Salud (oms).
- ICBF: el Instituto Colombiano de Bienestar Familiar (ICBF) fue identificado como fuente de información por once participantes y un participante identificó la página web de la entidad como medio de consulta regular.

- Secretarías: las diferentes secretarías de los gobiernos locales fueron identificadas como fuentes de información, entre ellas se mencionaron las de Salud, Educación y Acción Social.

Adicionalmente, se puede listar un grupo de instituciones, sistemas y bases de datos de orden nacional que son consultadas regularmente. En este listado se encontraron el Departamento Administrativo Nacional de Estadísticas (DANE), el Fondo de Solidaridad y Garantía (Fosyga), el Sistema Nacional de Vigilancia en Salud Pública (Sivigila) y algunos de sus subsistema como el Sistema de Vigilancia Epidemiológica de la Violencia Intrafamiliar, el Maltrato Infantil y la Violencia Sexual (Sivim), el Sistema de Vigilancia Epidemiológica de Sustancias Psicoactivas (Vespa) y el Sistema de Vigilancia Alimentaria y Nutricional (Sisvan), el Registro Único de Afiliados a la Protección Social (RUAF), el Sistema de Matrícula Estudiantil de Educación Básica y Media (Simat), el Ministerio de Salud y Protección Social (MSPS), la Base de datos Única de Afiliados (BDUA), el Departamento Nacional de Planeación (DNP), el Departamento para la Prosperidad Social (DPS), el Directorio Único de Establecimientos Educativos (DUE) y la Superintendencia Nacional de Salud (Supersalud).

\section{Principales inconvenientes}

Se construyeron dieciocho categorías de análisis que recogen los principales inconvenientes que se presentan en el momento de consultar la información. Las categorías más referenciadas fueron: "Falta de información/ 
información incompleta”, "Actualización de la información" y "Articulación/unificación y consolidación". La cuarta categoría más referenciada fue "Problemas de captura", donde se hace referencia al no registro correcto de la información, por ejemplo: cuando se digita el código de diagnóstico de la Clasificación Internacional de Enfermedades (CIE 10), que no corresponde al diagnóstico real del paciente; cuando las personas al dar sus datos de identificación encuentran que estos no son reales o cuando no se registra el nacimiento o muerte de los niños y niñas. Otro problema en la captura de la información se presenta cuando la población no denuncia diferentes casos de maltrato, abuso o violencia contra el menor, ocasionando no solo un subregistro de la información sino principalmente una falta de atención frente a la ocurrencia del evento. Por otro lado, en la categoría "No validez y confiabilidad", se incluyeron los inconvenientes relacionados con la veracidad de la información, es decir, que los participantes identifican como principal inconveniente que los datos o información que consultan no corresponde con la realidad del evento que están estudiando. Por su parte, dentro de la categoría "Problemas de socialización de la información" se agruparon los inconvenientes que se presentan al no conocer los medios a través de los cuales las personas pueden consultar la información, limitando el acceso a la población en general. La categoría "Falta de claridad en la fuente" hace referencia a que al consultar la información, las fuentes son muy extensas, se presentan en un lenguaje demasiado técnico o no son de fácil comprensión por parte de los participantes; se encontraron respuestas como "cómo la información apunta a las necesidades de la comunidad" o "los temas son demasiados extensos y en conclusión nada explican". En la categoría denominada "Fallas Internet/tecnología", el principal inconveniente fue la falta de acceso a internet y la velocidad en la que esta red opera en el territorio. También se mencionó en esta categoría la falta de herramientas tecnológicas o su mal funcionamiento, lo cual muestra la necesidad de equipos de cómputo apropiados y la falta de software o sistemas de monitoreo y evaluación para facilitar la consulta de la información y su procesamiento.

También se indagó acerca de los temas cuya información presenta deficiencias o no se encuentran disponibles. Como resultado se encontró que los temas que presentaron mayores deficiencias son los mismos que los participantes consideraron como los más importantes; estos son "Alimentación/estado nutricional", "Seguridad y protección" y "Educación". Otra categoría asociada con la presencia de inconvenientes al momento de la consulta es la correcta "Identificación y ubicación de la población”, donde se agrupan los datos de identificación y ubicación geográfica dentro del municipio y en particular se menciona a la "población flotante" "que ocasiona una alta variación en el número de habitantes en el municipio. Lo anterior tiene un impacto en la construcción de indicadores, que tienen como denominador de referencia la población proyectada por el DANE para el territorio. En el anexo 2 se presenta el listado de todas las categorías obtenidas en la parte individual del taller.

\section{Sobre los indicadores para la rendición pública de cuentas}

Se indagó sobre cuáles indicadores de los definidos para la rendición pública de cuentas (anexo 1 - pregunta 1 parte grupal) no miden de madera adecuada el CSDINN. Los participantes identificaron que los indicadores de las áreas temáticas "Educación”, "Juego” y

7 La población flotante se entiende como el grupo de habitantes que se desplazan entre municipios en ciertas temporadas a causa de su actividad económica, (p. ej. durante el cultivo de flores o época de cosechas de la cebolla). 
"Espacios sociales" son mediciones "muy generales" y no evalúan la calidad o logros alcanzados en estas actividades. También recalcaron que los indicadores cuyos resultados son número absoluto, como "Número de niños y niñas, entre 0 y 5 años, declaradas en situación de adoptabilidad" o "Número de niños y niñas, menores de 6 años, que asisten a bibliotecas con sus padres/acompañantes" no permiten realizar comparaciones o tomar decisiones sobre el evento de interés. Se indagó sobre cuáles de estos indicadores son los más difíciles de medir debido a problemas de información, y se encontró una situación particular relacionada con la población proyectada por el DANE, donde los participantes mencionan que esta proyección no corresponde a la realidad del municipio, lo cual afecta la medición de los indicadores incluidos en el área temática "Salud". También se identificaron dificultades de medición en los indicadores relacionados con casos de violencia infantil, los cuales presentan subregistro por el no reporte o denuncia de estos casos.

\section{Ventajas y desventajas de un nuevo sistema} de información. Entre las ventajas de contar con un sistema de información del CSDINN se identificaron: 1) contar con un sistema único en primera infancia, que integre las diferentes instituciones y redes de salud existentes; 2) permite tener un fácil acceso a información de calidad de manera oportuna; 3 ) permite formular nuevos programas en primera infancia, y a los ya existentes permite realizarles seguimiento y justificar o no su continuidad; 4) lograr identificar y ubicar geográficamente a la población objetivo; 5) identificar las principales necesidades del municipio para actuar en consecuencia; 6) al llegar alguien nuevo, este puede conocer fácilmente un panorama completo del municipio.
Por su parte, al indagar sobre desventajas, los participantes mencionaron aspectos que se deben considerar al momento de implementar el sistema. Estos aspectos fueron: 1) se requieren recursos tanto económicos como de personal por parte del municipio; 2) debe estar articulado con otros sistemas ya existentes; 3) las fuentes de información deben ser confiables; 4) el sistema debe tener un propósito claro; 5) debe tener continuidad; 6) debe contener todos los temas necesarios para los usuarios; 7) en las zonas rurales del territorio se presentan inconvenientes de implementación y captura de datos; 8) se puede hacer un uso inadecuado de la información.

\section{Aspectos o sugerencias para la construcción de un sistema de información}

Se indagó sobre tres elementos que se deben considerar en el diseño de un sistema: los usuarios, el área responsable de manejar el sistema y los responsables de reportar información a este y cómo realizarlo. Sobre el primer elemento los participantes concluyeron que este sistema debe estar habilitado a todos los actores que intervienen en el cuidado de la salud. En el segundo aspecto se mencionó a la secretaría de planeación municipal o departamental, y particularmente en el municipio de Tunja, el Observatorio Social, como las áreas encargadas de centralizar y consolidar la información. También en este punto los participantes recalcaron que el personal encargado de manejar el sistema debe estar capacitado o ser un "experto" y que se debe buscar su permanencia. En el tercer elemento fueron mencionadas las siguientes instituciones como las encargadas del reporte de información: ICBF, IPS, secretarías, comisarías de familia, Registraduría, Personería, Policía y padres. Con relación a cómo realizar el reporte de información, se sugirió a partir de informes o reporte mensuales o a través de un software. En este elemento, uno de los participantes mencionó que se debe conside- 
rar la sobrecarga de reportes ya existentes, citando como ejemplo a las IPS que responden por "34 informes mensuales y eso sin contar los informes de gestión".

Finalmente, se indagó sobre posibles indicadores que fuera preciso incluir dentro de un sistema de información de CSDINN, buscando que los participantes los asignaran a cada una de las cuatro categorías del cuidado de la salud propuestas por el programa IPV. Los participantes no realizaron definiciones de indicadores, pero sí mencionaron los temas que consideran fundamental incluir. En el anexo 3 se presenta el listado de temas mencionados por los participantes por cada una de las cuatro categorías.

\section{Discusión y conclusiones}

Una evaluación integral de la población infantil demanda el abordaje de diferentes áreas o temas enfocados a un objetivo claro, como lo realizan el Sistema Único de Información de la Niñez (suin) (16) y el Sistema de Información de Niñezy Adolescencia (Sinfonía) (10), donde a partir de las categorías de derecho y los objetivos de política se busca monitorizar a la garantía de los derechos de los niños y niñas, o cómo se realiza en la rendición pública de cuentas que deben realizar los territorios a la Procuraduría General de la Nación, donde a partir de áreas temáticas se busca hacer seguimiento al mismo objetivo. En esta investigación se identificaron varios temas (agrupados en 31 categorías), los cuales la mayoría se encuentran incluidos en los sistemas mencionados anteriormente, pero se pueden identificar cuatro categorías que no suelen ser consideradas en estos sistemas; estas: "Programas de promoción y prevención", "Corresponsabilidad de la familia", "Prácticas de cuidado" y "Redes y comunicación". Los temas incluidos en estas categorías deben ser tenidos en cuenta, bus- cando proponer indicadores que permitan su monitorización en los territorios. A este respecto, en un estudio realizado en Australia para identificar las áreas de prioridad para la salud y el bienestar de los niños de la ciudad de Victoria, ${ }^{8}$ se incluyeron los siguientes temas: ${ }^{9}$ obesidad y factores determinantes, bienestar y salud emocional y social, ambiente familiar, uso de los servicios de salud, enfermedades infantiles, comunidad, salud oral, lesiones, embarazo y lactancia y posición social (17). Asimismo, en el proyecto Kids Count ${ }^{10}$ se definieron dieciséis indicadores para medir el bienestar infantil, los cuales sirven para construir un índice del bienestar infantil (Kids Count Index), teniendo en cuenta los siguientes dominios: salud (física, mental y emocional), educación (logros), material/ económico, familia (ambiente /estructura / contexto), seguridad/comportamiento riesgoso y comunidad (18). Lo anterior permite observar que una visión más integral de lo que es necesario considerarse dentro del desarrollo integral y/o bienestar infantil involucra áreas adicionales a las convencionales y enfatiza en otras áreas que son determinantes en el desarrollo de los niños, aunque su medición requiera mayores esfuerzos en la recolección de información (17).

Con respecto a la identificación de fuentes de información relacionadas con el CSDINN,

8 En este estudio fueron realizadas 54 entrevistas a actores políticos y académicos, los cuales fueron seleccionados para abarcar un amplio rango de experiencia en áreas de salud pública y organizaciones inter-sectoriales relevantes en conseguir resultados en salud en los niños, incluyendo universidades, gobierno y agencias no gubernamentales a través de Victoria.

9 En este estudio se presentan los temas generales, las áreas y los datos específicos que son requeridos por las partes interesadas en esta temática.

10 Este proyecto comenzó en 1990, con tres objetivos principales. Por más de dos décadas ha usado más o menos los mismos diez indicadores para realizar el seguimiento del bienestar de los niños en el ámbito estatal. La última actualización del índice definió dieciséis indicadores para su construcción. 
en nuestro estudio se construyeron cincuenta categorías que agrupan estas fuentes. Lo anterior evidencia el gran esfuerzo que se debe realizar en un territorio para obtener una mirada integral de la situación de esta población. También se encontró que si bien existen diferentes sistemas de información en los territorios, las principales fuentes que son usualmente consultadas por los actores fueron las agrupadas en la categoría "Publicaciones/ informes/Documentos técnicos", la cual se caracteriza por agrupar fuentes de información que no permiten realizar un seguimiento de la población infantil de manera oportuna. Los dos hallazgos anteriores muestran la necesidad de contar con un espacio que permita a los diferentes actores obtener un panorama completo de la población infantil donde se articulen las diferentes instituciones y se consoliden de forma continua las evaluaciones sobre los diferentes temas de interés.

Ante el gran número de fuentes de información que se presenta en los territorios, de igual forma existe un gran número de inconvenientes que hacen referencia principalmente a la falta de articulación, unificación y consolidación de la información. Lo anterior evidencia que no existen procesos que estandaricen un flujo constante de la información, ocasionando que los actores en los territorios deban tomar decisiones a partir de resultados desactualizados, incompletos o muchas veces inexistentes. Ante la carencia de estos procesos, los actores empiezan a desconfiar de la veracidad de la información que reciben, lo cual fue mencionado por varios de ellos, al decir que esta no representa la realidad de sus municipios. La anterior situación presenta mayor gravedad cuando al identificar los temas donde se presentan los mayores inconvenientes, estos coinciden con los que ellos mismos identifican como los más importantes. Estos hallazgos concuerdan con un estudio realizado por la OMS ${ }^{11}$ (19) el cual concluyó que la baja calidad de los datos ha sido identificada como uno de los mayores problemas que afectan la utilidad de los datos de las instalaciones de salud para la toma de decisiones locales y nacionales y, asimismo, que la capacidad analítica de la información es usualmente marcada como una de las mayores debilidades en países con ingresos bajos y medios. La oms (19) también sostiene que el acceso público a la salud es esencial para mejorar la rendición de cuentas; adicionalmente, el compartir datos entre las diferentes entidades que manejan información en la materia, facilita la compilación de estadísticas confiables, las cuales pueden convertirse en la base de la promoción, la política y la acción efectiva.

Una necesidad puntual que surge en el territorio se relaciona con los diferentes programas o estrategias que apuntan a la población infantil y que actualmente se implementan en los municipios, y es la de evaluar si ellas están alcanzando el impacto esperado, si la población beneficiada cumple los criterios necesarios para acceder al programa o estrategia y si efectivamente está dirigida a solucionar la problemática real del municipio. Por otra parte, uno de los principales retos que enfrentaría el diseño de un sistema de información es lograr la identificación de cada uno de los niños y niñas menores de seis años, al igual que poder realizarle un seguimiento a través de las diferentes áreas de cuidado de la salud. Cubrir esta necesidad le permitiría al gobierno local contrastar su densidad poblacional con las proyecciones del DANE, ya que en varias ocasiones se mencionó que estas proyecciones no se ajustan a la realidad de sus territorios. Este reto se incrementa aún más cuando el interés no es únicamente identificar a la población dentro

11 Este reporte tiene como objetivo proveer una vista general del estado de los sistemas de información en países de ingresos bajos y medianos. 
de su territorio, sino también cuándo esta se desplaza a otros municipios, ya sea a recibir diferentes servicios o porque su movilidad se ve asociada a la actividad económica que realiza el jefe cabeza de familia (población flotante). Para cubrir esta necesidad se requiere, además de la disponibilidad de recursos humanos y financieros, la integración y articulación de todos los actores que intervienen en el cuidado de la salud de la población infantil en los distintos territorios.

Finalmente, dos aspectos que se deben considerar en el diseño de un sistema de información relacionado con el CSDINN y que se implemente en los municipios, son: i) los diferentes sistemas o herramientas que ya existen en el territorio, los cuales no pueden ser reemplazados o modificados, y ii) los requerimientos de reporte de información que deben cumplir los actores locales ante las entidades del nivel nacional y departamental. Con relación al primer aspecto, el no considerar las herramientas o sistemas ya existentes en los territorios ocasionaría una sobrecarga de trabajo, pues daría lugar a un nuevo proceso de captura de información, perjudicando con ello aspectos claves como la validez, la confiabilidad y la oportunidad. Sobre el segundo aspecto, el no considerar los requerimientos de reporte de información que deben realizar los actores ocasionaría que el sistema no se apropie o no se utilice si este no les presenta un claro beneficio. El sistema debe facilitar la obtención, el procesamiento y la presentación de resultados, permitiendo a los actores dedicar mayor tiempo al análisis y discusión de la situación que se presenta en la población infantil en su municipio.

\section{Referencias bibliográficas}

1. Programa de las Naciones Unidas para el Desarro1lo. Informe sobre desarrollo humano 2003. Los objetivos de desarrollo del milenio: Un pacto entre las naciones para eliminar la pobreza. Nueva York: Ediciones Mundi-Prensa; 2003.

2. Ministerio de la Protección Social. Plan Nacional para la Niñez y la Adolescencia 2009-2019. Bogotá: Imprenta Nacional; 2009.

3. Harrison T, Nutley T. A Review of Constraints to Using Data for Decision Making: Recommendations to Inform the Design of Interventions. Chapel Hill, NC: Measure Evaluation, Carolina Population Center; 2010.

4. Health Metrics Network. Framework and Standards for Country Health Information Systems. Ginebra: World Health Organization; 2008.

5. World Health Organization. WHOsIs. wHO Statistical Information System. Ginebra: World Health Organization; 2013 [Internet] [acceso: 11 de diciembre del 2013]. Disponible en: http://apps.who.int/ whosis/data/Search.jsp.

6. Siteal. Sistema de Información para la Primera Infancia en América Latina SIPI. Buenos Aires: Siteal; 2013 [Internet] [acceso: 11 de diciembre del 2013]. Disponible en http://www.sipi.siteal.org

7. Ministerio de Salud y Protección Social. Registros, observatorios, sistemas de seguimiento y salas situacionales nacionales. Sistema de Monitoreo y Evaluación al Plan Decenal de Salud Pública 20122021. Bogotá: Ministerio de Salud y Protección Social; 2013.

8. Estrategia Hechos y Derechos. Proceso de rendición pública de cuentas sobre la gestión para la garantía de los derechos de la infancia, la adolescencia y la juventud. Indicadores sociales y gasto público social. Bogotá: Calle Impresores; 2011.

9. Mesa Sistema Único de Información de la Niñez. Revista SUIN Sistema Único de Información de la Niñez. Bogotá D.C.: Carvajal Soluciones de Comunicación; 2012.

10. Unicef Colombia. Sistema de Información de Niñez y Adolescencia - Sinfonía. Bogotá: Unicef; 2013 [Internet] [acceso: 11 de diciembre del 2013]. Disponible en http://www.sinfoniaunicef.info/

11. Rodríguez González H, Rueda Ramírez C. Sistema de información para la vigilancia en salud pública: Propuesta conceptual y tecnológica. Bogotá: Organización Panamericana de la Salud, Ministerio de la Protección Social; 2005 [Internet] [acceso: 11 de diciembre del 2013]. Disponible en http://www.ins. gov.co/lineas-de-accion/Subdireccion-Vigilancia/ sivigila/Documentos $\% 20$ SIVIGILA/Sistema $\% 20$ de\%20Informacion\%20para\%20la\%20VSP.pdf

12. Ministerio de Salud y Protección Social. Sispro Sistema Integral de Información de la Protección Social en Salud. Bogotá: Ministerio de Salud y Protección Social; 2013 [Internet] [acceso: $30 \mathrm{de}$ noviembre del 2013]. Disponible en http://www. sispro.gov.co/

13. Departamento Administrativo Nacional de Estadísticas. Estadísticas vitales. Colombia 2013 [Internet] [acceso: 11 de diciembre del 2011]. Disponible en http://www.dane.gov.co/index.php/poblacion-ydemografia/nacimientos-y-defunciones.

14. Departamento Administrativo Nacional de Estadística. Proyecciones de población. Colombia 
2013 [Internet] [acceso: 11 de diciembre de 2013]. Disponible en http://www.dane.gov.co/index. php/poblacion-y-demografia/proyecciones-depoblacion.

15. Nutley T, Reynolds HW. Improving the Use of Health Data for Health System Strengthening. Glob Health Action. 2013; (6).

16. Instituto Colombiano de Bienestar Familiar. Sistema Único de Información de la Niñez suIN. Colombia 2013 [Internet] [acceso: 11 de diciembre del 2013]. Disponible en: http://www.suin-snbf.gov. co/suin/Pages/Porindicador.aspx.
17. Davis E, Walters E, Wake M, Goldfeld S, Williams J, Mehmet-Radji $\mathrm{O}$ et al. Population Health and Wellbeing: Identifying Priority Areas for Victorian Children. Australia and New Zealand Health Policy. 2005 Jul 20; 16 (2).

18. The Annie E. Casey Foundation. The New Kids Count Index. Baltimore: The Annie E. Casey Foundation; 2012.

19. World Health Organization. Country Health Information Systems: A Review of the Current Situation and Trends. Suiza: World Health Organization; 2011.

\section{Anexo 1}

Instrumento

\section{Actividad individual}

1. ¿Cuáles son los temas relacionados con el CSDINN que son de principal interés para la entidad que usted representa?

2. ¿Cuáles son las principales fuentes de información relacionadas con temas del CSDINN que se consultan regularmente en la entidad a la que usted representa?

3. ¿Cuáles son los cinco (5) principales inconvenientes que tiene en el momento de consultar las fuentes de información?

4. ¿En qué temas relacionados con el CSDINN, y que usted considere relevantes, la información documental y estadística es deficiente o no se encuentra disponible? 
5. Priorice el orden de importancia de los siguientes niveles de desagregación de la información al momento de realizar análisis sobre los temas relacionados con el CSDINN, siendo el 1 el de mayor importancia y el 14 el de menor.

\begin{tabular}{|l|l|}
\hline Periodicidad & \\
\hline Grupos de edad & \\
\hline Sexo & \\
\hline Zona (urbano- rural) & \\
\hline Ubicación (departamento) & \\
\hline Ubicación (municipio) & \\
\hline Ubicación (barrio / vereda) & \\
\hline Estratificación & \\
\hline
\end{tabular}

\begin{tabular}{|c|c|}
\hline Nivel educativo & \\
\hline Acceso a servicios públicos & \\
\hline Tipo de afiliación al sGsss & \\
\hline Participación en programas sociales & \\
\hline $\begin{array}{l}\text { Características socioeconómicas } \\
\text { del grupo familiar }\end{array}$ & \\
\hline Otro & \\
\hline
\end{tabular}

En el caso de otro, indique cual:

6. De los medios de información listados a continuación, seleccione con una X los tres (3) que más utiliza para realizar la consulta de información relacionada con csDinN.

\begin{tabular}{|l|l|}
\hline Páginas web & \\
\hline Cartillas & \\
\hline Televisión & \\
\hline Estudios & \\
\hline Periódicos & \\
\hline Normatividad & \\
\hline Charlas y conferencias & \\
\hline
\end{tabular}

\begin{tabular}{|l|l|}
\hline Revistas & \\
\hline Informes técnicos o reportes institucionales & \\
\hline Estadísticas institucionales & \\
\hline Bases de datos institucionales & \\
\hline Informes o publicaciones institucionales & \\
\hline Otros & \\
\hline & \\
\hline
\end{tabular}

7. Enumere tres (3) medios a través de los cuales le gustaría consultar información (documental y estadística) y explique por qué.

\begin{tabular}{|l|l|l|}
\hline & Medio de información & ¿Por qué? \\
\hline 1 & & \\
\hline 2 & & \\
\hline 3 & & \\
\hline
\end{tabular}




\section{Actividad grupal}

1. De la lista de los 65 indicadores priorizados, y que están relacionados con el CSDSNN, responda las siguientes preguntas: a) ¿Cuáles indicadores considera que no miden de manera adecuada csDinn? b) ¿Cuáles indicadores son los más difíciles de medir debido a problemas de información? (el listado de indicadores se incluye al final del instrumento).

2. De acuerdo con su opinión, ¿Cuáles considera son las ventajas y desventajas de la existencia de un sistema de información del CSDINN que opere a nivel departamental y municipal?

3. ¿Qué elementos considera deberían incluirse en el diseño de un sistema de información del CSDINn, que opere para el departamento y sus municipios, y que consolide la información de todas las instituciones participantes? Para su respuesta considere los siguientes aspectos: ¿Quiénes serían los principales usuarios?, ¿quién debería manejar el sistema de información?, y iquiénes y cómo se debería reportar la información?

4. ¿Qué indicadores considera usted que debería(n) incluirse dentro del Sistema de Información de CSDinn que evalúen los cuatro componentes del programa? Preparación para la vida, Prácticas cotidianas para la vida, Seguridad e integridad corporal y Educación inicial.

\section{Listado de indicadores pregunta \# 1- Actividad grupal}

\begin{tabular}{|c|c|c|}
\hline \multicolumn{3}{|l|}{ Área temática: mortalidad } \\
\hline 1. Razón de mortalidad materna & $\begin{array}{l}\text { 3. Tasa de mortalidad de niños y } \\
\text { niñas entre los } 0 \text { a } 5 \text { años }\end{array}$ & $\begin{array}{l}\text { 5. Tasa de mortalidad de niños y } \\
\text { niñas, entre } 0 \text { a } 5 \text { años, por causas } \\
\text { externas (homicidio, suicidio, ac- } \\
\text { cidentes, violencia intrafamiliar) }\end{array}$ \\
\hline $\begin{array}{l}\text { 2. Tasa de mortalidad en menores } \\
\text { de } 1 \text { año - mortalidad infantil }\end{array}$ & $\begin{array}{l}\text { 4. Cinco primeras causas de } \\
\text { mortalidad de niños y niñas entre } \\
\text { los } 0 \text { y } 5 \text { años }\end{array}$ & \\
\hline \multicolumn{3}{|l|}{ Área temática. Nutrición } \\
\hline $\begin{array}{l}\text { 6. Porcentaje de niños y niñas } \\
\text { menores de } 6 \text { años con desnutri- } \\
\text { ción crónica }\end{array}$ & $\begin{array}{l}\text { 8. Porcentaje de niños, niñas } \\
\text { entre } 0-6 \text { meses que asisten a } \\
\text { controles de crecimiento y de- } \\
\text { sarrollo y que reciben lactancia } \\
\text { materna exclusiva }\end{array}$ & $\begin{array}{l}\text { 10. Porcentaje de niños, niñas con } \\
\text { bajo peso al nacer }\end{array}$ \\
\hline $\begin{array}{l}\text { 7. Porcentaje de niños y niñas } \\
\text { menores de } 6 \text { años valorados con } \\
\text { desnutrición global }\end{array}$ & $\begin{array}{l}\text { 9. Porcentaje de mujeres gestan- } \\
\text { tes con diagnóstico de anemia } \\
\text { nutricional }\end{array}$ & \\
\hline \multicolumn{3}{|l|}{ Área temática: salud } \\
\hline $\begin{array}{l}\text { 11. Cobertura de inmunización } \\
\text { contra el BCG en niños y niñas } \\
\text { menores de un año }\end{array}$ & $\begin{array}{l}\text { 18. Cobertura de inmunización } \\
\text { contra la influenza en niños y } \\
\text { niñas menores de } 1 \text { año }\end{array}$ & $\begin{array}{l}\text { 24. Tasa de morbilidad por EDA } \\
\text { (enfermedad diarreica aguda) en } \\
\text { menores de } 6 \text { años }\end{array}$ \\
\hline
\end{tabular}


12. Cobertura de inmunización contra el polio en niños y niñas menores de un año

13. Cobertura de inmunización contra el DPT en niños y niñas menores de 1 año

14. Cobertura de inmunización contra la Hepatitis B en niños y niñas menores de 1 año

15. Cobertura de inmunización contra el rotavirus en niños y niñas menores de 1 año

16. Cobertura de inmunización contra el neumococo en niños y niñas de 1 año

17. Cobertura de inmunización contra la triple viral en niños y niñas de un año
19. Porcentaje de mujeres gestantes que asistieron a control prenatal y que se practicaron la prueba de VIH (Elisa)

20. Tasa de transmisión materno infantil de VIH

21. Porcentaje de embarazos en mujeres adolescentes

22. Porcentaje de mujeres gestantes con sífilis que han sido diagnosticadas y tratadas antes de la semana 17

65. Tasa de sífilis congénita

23. Cinco primeras causas de morbilidad en menores de 6 años
25. Tasa de morbilidad por ERA (enfermedad respiratoria aguda) en menores de 6 años

26. Porcentaje de niños, niñas entre 0 y 5 años que asisten a controles de crecimiento y desarrollo

27. Cobertura de agua

28. Cobertura de saneamiento básico

29. Cobertura con agua potable

Área temática: familia

30. Número de niños y niñas entre 0 y 5 años declarados en situación de adoptabilidad
31. Porcentaje de niños y niñas entre 0 y 5 años declarados adoptables y dados en adopción
32. Número estimado de personas menores de 6 años en situación de calle

Área temática: educación

33. Porcentaje de niños y niñas vinculados a programas de educación inicial

Área temática. Juego

44. Número de niños y niñas menores de 6 años que asisten a bibliotecas con sus padres/acompañantes

45.1. Porcentaje de niños y niñas menores de 6 años matriculados o inscritos en programas de recreación y deporte, festivales escolares

Área temática: espacios sociales

48. Porcentaje de gobiernos escolares operando

45.2. Porcentaje de niños y niñas menores de 6 años matriculados o inscritos en programas de recreación y deporte - escuelas de formación deportiva
46. Porcentaje de niños y niñas menores de 6 años inscritos o matriculados en programas artísticos, lúdicos o culturales

49. Porcentaje de consejos de política social (departamental y municipales) en los que participan niños y niñas

Área temática: registro

51. Proporción de niños y niñas menores de 1 año registrados según lugar de nacimiento

Área temática: abuso y conflicto interno

55. Tasa de informes periciales sexológicos en menores de 6 años

58. Porcentaje de niños y niñas menores de 6 años desplazados por la violencia 
53. Número de casos de denuncia por abuso sexual en niños y niñas menores de 6 años

54. Número de casos de informes periciales sexológicos en menores de 6 años
56. Número de valoraciones médico-legales por presunto delito de maltrato infantil

57. Porcentaje de niños y niñas menores de 6 años que son víctimas de minas antipersona y municiones sin explotar

\section{Área temática: actividades perjudiciales}

59. Número de niños y niñas meno- 60 . Número de niños y niñas menores de 6 años que participan en una actividad remunerada o no res de 6 años que trabajan 15 o más horas en oficios del hogar
61. Número de niños y niñas menores de 6 años explotados sexualmente

Fuente: elaboración propia adaptada del Diagnóstico e Informe Situacional de Infancia y Adolescencia de la Procuraduría General de la Nación

\section{Anexo 2}

Listado de categorías obtenidas en la parte individual del taller

\begin{tabular}{|c|c|c|c|c|c|}
\hline \multicolumn{2}{|c|}{ Temas de mayor interés } & \multicolumn{2}{|c|}{ Fuentes de información } & \multirow{2}{*}{\begin{tabular}{l}
\multicolumn{1}{c}{ Principales } \\
inconvenientes \\
al momento de \\
\multicolumn{1}{c}{ la consulta } \\
Falta in- \\
formación/ \\
información \\
incompleta \\
$(26)$
\end{tabular}} & \multirow{2}{*}{$\begin{array}{l}\begin{array}{c}\text { Temas que } \\
\text { presentan }\end{array} \\
\text { mayores incon- } \\
\text { venientes }\end{array}$} \\
\hline $\begin{array}{l}\text { Prácticas coti- } \\
\text { dianas para el } \\
\text { cuidado de la } \\
\text { salud }\end{array}$ & $\begin{array}{l}\text { Educación } \\
\text { inicial }\end{array}$ & $\begin{array}{l}\text { Publicacio- } \\
\text { nes/infomes/ } \\
\text { documentos } \\
\text { técnicos (20) }\end{array}$ & $\begin{array}{l}\text { Inspección de } \\
\text { policía (2) }\end{array}$ & & \\
\hline $\begin{array}{l}\text { Alimentación/ } \\
\text { estado nutri- } \\
\text { cional }(27) *\end{array}$ & Educación (10) & $\begin{array}{l}\text { Estrategias/ } \\
\text { programas (13) }\end{array}$ & $\begin{array}{l}\text { Vacunación - } \\
\text { MinSalud (2) }\end{array}$ & $\begin{array}{l}\text { Actualización } \\
\text { de la informa- } \\
\text { ción }(25)\end{array}$ & $\begin{array}{l}\text { Alimentación/ } \\
\text { estado nutri- } \\
\text { cional (9) }\end{array}$ \\
\hline Desarrollo (18) & $\begin{array}{l}\text { Cultura, } \\
\text { recreación y } \\
\text { deporte (7) }\end{array}$ & ESE/IPS (13) & $\begin{array}{l}\text { Internet/pági- } \\
\text { nas web - alcal- } \\
\text { día municipal } \\
\text { (2) }\end{array}$ & $\begin{array}{l}\text { Articulación/ } \\
\text { unificación y } \\
\text { consolidación } \\
\text { (21) }\end{array}$ & Salud (6) \\
\hline $\begin{array}{l}\text { Programas de } \\
\text { promoción y } \\
\text { prevención } \\
\text { (16) }\end{array}$ & $\begin{array}{l}\text { Estimulación } \\
\text { (2) }\end{array}$ & Sisben (12) & $\begin{array}{l}\text { internet/pági- } \\
\text { nas web - ICBF } \\
\text { (1) }\end{array}$ & $\begin{array}{l}\text { Problemas en } \\
\text { la captura (18) }\end{array}$ & $\begin{array}{l}\text { Prácticas de } \\
\text { cuidado (2) }\end{array}$ \\
\hline $\begin{array}{l}\text { Corresponsa- } \\
\text { bilidad de la } \\
\text { familia (14) }\end{array}$ & $\begin{array}{l}\text { Proyecto de } \\
\text { vida (1) }\end{array}$ & $\begin{array}{l}\text { Normas/leyes/ } \\
\text { lineamientos/ } \\
\text { resoluciones } \\
\text { (11) }\end{array}$ & Sicresub (1) & $\begin{array}{l}\text { No validez ni } \\
\text { confiabilidad } \\
\text { (15) }\end{array}$ & Desarrollo (2) \\
\hline $\begin{array}{l}\text { Prácticas de } \\
\text { cuidado (10) }\end{array}$ & Otros & ICBF (11) & $\begin{array}{l}\text { Unidad de dis- } \\
\text { capacidad (1) }\end{array}$ & $\begin{array}{l}\text { Problemas de } \\
\text { socialización } \\
\text { de la informa- } \\
\text { ción (13) }\end{array}$ & $\begin{array}{l}\text { Programas de } \\
\text { promoción y } \\
\text { prevención (2) }\end{array}$ \\
\hline $\begin{array}{l}\text { Crecimiento } \\
\text { (10) }\end{array}$ & $\begin{array}{l}\text { Estrategias o } \\
\text { programas (9) }\end{array}$ & $\begin{array}{l}\text { Secretarías } \\
\text { (11) }\end{array}$ & $\begin{array}{l}\text { Coordinación } \\
\text { primera infan- } \\
\text { cia (1) }\end{array}$ & $\begin{array}{l}\text { Falta de } \\
\text { claridad en la } \\
\text { fuente (12) }\end{array}$ & $\begin{array}{l}\text { Crecimiento } \\
\text { (2) }\end{array}$ \\
\hline
\end{tabular}




\begin{tabular}{|c|c|c|c|c|c|}
\hline \multicolumn{2}{|c|}{ Temas de mayor interés } & \multicolumn{2}{|c|}{ Fuentes de información } & $\begin{array}{c}\text { Principales } \\
\text { inconvenientes } \\
\text { al momento de } \\
\text { la consulta }\end{array}$ & $\begin{array}{c}\text { Temas que } \\
\text { presentan } \\
\text { mayores incon- } \\
\text { venientes }\end{array}$ \\
\hline $\begin{array}{l}\text { Vacunación } \\
\text { (10) }\end{array}$ & $\begin{array}{l}\text { Entorno de } \\
\text { política }(6)\end{array}$ & $\begin{array}{l}\text { Fuente prima- } \\
\text { ria población } \\
\text { objetivo }(9)\end{array}$ & Alcaldía (1) & $\begin{array}{l}\text { Fallas internet/ } \\
\text { tecnología (12) }\end{array}$ & $\begin{array}{l}\text { Pautas de } \\
\text { crianza (2) }\end{array}$ \\
\hline Salud (9) & $\begin{array}{l}\text { Difusión y } \\
\text { participación } \\
\text { del programa } \\
\text { CSDINN (4) }\end{array}$ & $\begin{array}{l}\text { Espacios de } \\
\text { capacitación } \\
(8)\end{array}$ & BDUA (1) & $\begin{array}{l}\text { Falta de } \\
\text { oportunidad de } \\
\text { la información } \\
\text { (11) }\end{array}$ & $\begin{array}{l}\text { Eventos de } \\
\text { interés en salud } \\
\text { pública (2) }\end{array}$ \\
\hline $\begin{array}{l}\text { Eventos de } \\
\text { interés en salud } \\
\text { pública (6) }\end{array}$ & $\begin{array}{l}\text { Entidades o } \\
\text { instituciones } \\
\text { (3) }\end{array}$ & $\begin{array}{l}\text { Internet/pági- } \\
\text { nas web (8) }\end{array}$ & $\begin{array}{l}\text { Superintenden- } \\
\text { cia de Salud (1) }\end{array}$ & $\begin{array}{l}\text { Falta de capaci- } \\
\text { tación (9) }\end{array}$ & $\begin{array}{l}\text { Corresponsa- } \\
\text { bilidad de la } \\
\text { familia (1) }\end{array}$ \\
\hline $\begin{array}{l}\text { Pautas de } \\
\text { crianza (6) }\end{array}$ & & $\begin{array}{l}\text { Medios de } \\
\text { comunicación } \\
\text { (7) }\end{array}$ & $\begin{array}{l}\text { Oficina el Nue- } \\
\text { vo Ciudadano } \\
\text { Boyacense (1) }\end{array}$ & $\begin{array}{l}\text { Dificultades } \\
\text { en el acceso a } \\
\text { la información } \\
\text { (7) }\end{array}$ & $\begin{array}{l}\text { Seguridad e } \\
\text { integridad } \\
\text { corporal }\end{array}$ \\
\hline Salud oral (5) & & $\begin{array}{l}\text { Fuentes } \\
\text { propias del } \\
\text { municipio (6) }\end{array}$ & Vespa (1) & $\begin{array}{l}\text { Falta de com- } \\
\text { promiso (6) }\end{array}$ & $\begin{array}{l}\text { Seguridad/pro- } \\
\text { tección }(12)\end{array}$ \\
\hline $\begin{array}{l}\text { Entornos salu- } \\
\text { dables (2) }\end{array}$ & & DANE (5) & $\begin{array}{l}\text { Procuraduría } \\
\text { (1) }\end{array}$ & $\begin{array}{l}\text { Voluntad } \\
\text { política (6) }\end{array}$ & $\begin{array}{l}\text { Identificación y } \\
\text { ubicación de la } \\
\text { población (9) }\end{array}$ \\
\hline Valores (1) & & Fosyga (5) & $\begin{array}{l}\text { Defensoría } \\
\text { Pública (1) }\end{array}$ & $\begin{array}{l}\text { No sistema- } \\
\text { tización de la } \\
\text { información } \\
\text { (5) }\end{array}$ & $\begin{array}{l}\text { Afiliación/acce- } \\
\text { so/atención al } \\
\text { SGSSS (7) }\end{array}$ \\
\hline $\begin{array}{l}\text { Preparación } \\
\text { para la vida }\end{array}$ & & Sivigila (5) & $\begin{array}{l}\text { Internet/pági- } \\
\text { nas web - Fosy- } \\
\text { ga (1) }\end{array}$ & $\begin{array}{l}\text { Falta de tiem- } \\
\text { po y exceso de } \\
\text { trabajo (4) }\end{array}$ & $\begin{array}{l}\text { Condiciones } \\
\text { sociodemográ- } \\
\text { ficas (5) }\end{array}$ \\
\hline $\begin{array}{l}\text { Cuidado de la } \\
\text { gestante/lac- } \\
\text { tante (9) }\end{array}$ & & $\begin{array}{l}\text { Base de datos } \\
\text { Aseguramiento } \\
\text { (5) }\end{array}$ & $\begin{array}{l}\text { Representante } \\
\text { de seguridad } \\
\text { alimentaria } \\
\text { (gobernación) } \\
\text { (1) }\end{array}$ & $\begin{array}{l}\text { Falta de segui- } \\
\text { miento (2) }\end{array}$ & $\begin{array}{l}\text { Salud sexual y } \\
\text { reproductiva } \\
\text { (1) }\end{array}$ \\
\hline $\begin{array}{l}\text { Seguridad e } \\
\text { integridad } \\
\text { corporal }\end{array}$ & & $\begin{array}{l}\text { Comisaria de } \\
\text { familia (4) }\end{array}$ & $\begin{array}{l}\text { Internet/ } \\
\text { Páginas web - } \\
\text { Ministerio de } \\
\text { Salud (1) }\end{array}$ & $\begin{array}{l}\text { Tramitomanía } \\
\text { (1) }\end{array}$ & $\begin{array}{l}\text { Discapacidad } \\
\text { (1) }\end{array}$ \\
\hline $\begin{array}{l}\text { Seguridad/pro- } \\
\text { tección (23) }\end{array}$ & & Sisvan (4) & Biblioteca (1) & Ninguno (1) & $\begin{array}{l}\text { Salud mental } \\
\text { (1) }\end{array}$ \\
\hline $\begin{array}{l}\text { Afiliación/acce- } \\
\text { so/atención al } \\
\text { SGSSS (13) }\end{array}$ & & $\begin{array}{l}\text { Departamento } \\
\text { de Salud Públi- } \\
\text { ca (4) }\end{array}$ & $\begin{array}{l}\text { Unidad de } \\
\text { Atención } \\
\text { Integral (1) }\end{array}$ & & $\begin{array}{l}\text { Educación } \\
\text { inicial }\end{array}$ \\
\hline $\begin{array}{l}\text { Redes y comu- } \\
\text { nicación (7) }\end{array}$ & & $\begin{array}{l}\text { Instituciones } \\
\text { educativas (4) }\end{array}$ & $\begin{array}{l}\text { Departamento } \\
\text { para la Prospe- } \\
\text { ridad Social (1) }\end{array}$ & & Educación (8) \\
\hline $\begin{array}{l}\text { Condiciones } \\
\text { socio demográ- } \\
\text { ficas ( } 7 \text { ) }\end{array}$ & & RUAF (3) & $\begin{array}{l}\text { Base de datos } \\
\text { DNP (1) }\end{array}$ & & $\begin{array}{l}\text { Cultura, } \\
\text { recreación y } \\
\text { deporte (2) }\end{array}$ \\
\hline
\end{tabular}




\begin{tabular}{|c|c|c|c|c|}
\hline Temas de mayor interés & \multicolumn{2}{|c|}{ Fuentes de información } & $\begin{array}{c}\text { Principales } \\
\text { inconvenientes } \\
\text { al momento de } \\
\text { la consulta }\end{array}$ & $\begin{array}{l}\text { Temas que } \\
\text { presentan } \\
\text { mayores incon- } \\
\text { venientes }\end{array}$ \\
\hline $\begin{array}{l}\text { Discapacidad } \\
\text { (5) }\end{array}$ & RIPS (3) & $\begin{array}{l}\text { Directorio } \\
\text { único de } \\
\text { establecimien- } \\
\text { tos educativos } \\
\text { (DUE) (1) }\end{array}$ & & $\begin{array}{l}\text { Estimulación } \\
\text { (1) }\end{array}$ \\
\hline Adicciones (4) & Simat (3) & $\begin{array}{l}\text { Consejo de } \\
\text { política social } \\
\text { (1) }\end{array}$ & & Otros \\
\hline $\begin{array}{l}\text { Salud sexual y } \\
\text { reproductiva } \\
\text { (3) }\end{array}$ & Sicaps (2) & $\begin{array}{l}\text { Centros amiga- } \\
\text { bles (1) }\end{array}$ & & $\begin{array}{l}\text { Estrategias o } \\
\text { programas (8) }\end{array}$ \\
\hline $\begin{array}{l}\text { Salud mental } \\
\text { (3) }\end{array}$ & EPS (2) & & & $\begin{array}{l}\text { Los temas rela- } \\
\text { cionados con el } \\
\text { diagnóstico del } \\
\text { municipio (3) }\end{array}$ \\
\hline $\begin{array}{l}\text { Identificación y } \\
\text { ubicación de la } \\
\text { población (1) }\end{array}$ & Sivim (2) & & & $\begin{array}{l}\text { Entidades o } \\
\text { instituciones } \\
\text { (2) }\end{array}$ \\
\hline & & & & $\begin{array}{l}\text { Normas/leyes/ } \\
\text { lineamientos/ } \\
\text { resoluciones } \\
\text { (1) }\end{array}$ \\
\hline
\end{tabular}

* Los valores entre paréntesis () corresponden al número de veces que fue referenciada la categoría por los participantes.

Fuente: elaboración propia con base en los resultados de la investigación

\section{Anexo 3}

Listado de temas relacionados con el CSDINN sugeridos por los participantes

\begin{tabular}{|l|l|l|l|l|}
\hline $\begin{array}{l}\text { Prácticas cotidi- } \\
\text { anas para el cui- } \\
\text { dado de la salud }\end{array}$ & $\begin{array}{l}\text { Preparación para } \\
\text { la vida }\end{array}$ & $\begin{array}{l}\text { Seguridad e integ- } \\
\text { ridad corporal }\end{array}$ & Educación inicial & Otros indicadores \\
\hline $\begin{array}{l}\text { Nivel de educación } \\
\text { de los cuidadores }\end{array}$ & $\begin{array}{l}\text { Información que } \\
\text { recibe la madre en } \\
\text { los primeros años } \\
\text { de vida }\end{array}$ & $\begin{array}{l}\text { Garantizar el } \\
\text { derecho a la salud y deber }\end{array}$ & $\begin{array}{l}\text { Acceso a educación } \\
\text { inicial (hogares } \\
\text { comunitarios y jar- } \\
\text { dines infantiles) }\end{array}$ & $\begin{array}{l}\text { Tipo de trabajo que } \\
\text { realizan los niños de } 6 \text { años }\end{array}$ \\
\hline
\end{tabular}




\begin{tabular}{|c|c|c|c|c|}
\hline $\begin{array}{l}\text { Prácticas cotidi- } \\
\text { anas para el cui- } \\
\text { dado de la salud }\end{array}$ & $\begin{array}{l}\text { Preparación para } \\
\text { la vida }\end{array}$ & $\begin{array}{l}\text { Seguridad e integ- } \\
\text { ridad corporal }\end{array}$ & Educación inicial & Otros indicadores \\
\hline $\begin{array}{l}\text { Desarrollo desde } \\
\text { el punto de vista } \\
\text { cultural. Tener en } \\
\text { cuenta la diversidad } \\
\text { cultural, tradiciones } \\
\text { y región de proce- } \\
\text { dencia }\end{array}$ & $\begin{array}{l}\text { Información que re- } \\
\text { cibe antes y durante } \\
\text { la adolescencia }\end{array}$ & Registro de niños & $\begin{array}{l}\text { Facilidad de acceso } \\
\text { y ampliar cober- } \\
\text { turas }\end{array}$ & $\begin{array}{l}\text { Causas de trabajo } \\
\text { de estos niños }\end{array}$ \\
\hline $\begin{array}{l}\text { Maltrato entre ni- } \\
\text { ños en los colegios } \\
\text { (matoneo) }\end{array}$ & $\begin{array}{l}\text { Prevención de em- } \\
\text { barazos prematuros }\end{array}$ & $\begin{array}{l}\text { Programas de } \\
\text { estimulación motriz } \\
\text { temprana }\end{array}$ & $\begin{array}{l}\text { Crear espacios de } \\
\text { estimulación adec- } \\
\text { uada con enfoque } \\
\text { de } 0 \text { a } 5 \text { años para } \\
\text { su buen desarrollo } \\
\text { a nivel de todas } \\
\text { sus áreas (físico, } \\
\text { cognitivo, social) }\end{array}$ & $\begin{array}{l}\text { Quiénes acompa- } \\
\text { ñan a los niños }\end{array}$ \\
\hline Valores infundidos & $\begin{array}{l}\text { Formas de planifi- } \\
\text { cación }\end{array}$ & $\begin{array}{l}\text { Generación de } \\
\text { espacios recre- } \\
\text { ativos con el fin } \\
\text { de motivar a los } \\
\text { niños y niña en } \\
\text { el conocimiento, } \\
\text { cuidado y respeto } \\
\text { por su cuerpo a } \\
\text { nivel individual y } \\
\text { su entorno }\end{array}$ & $\begin{array}{l}\text { Estimulación tem- } \\
\text { prana y educación a } \\
\text { padres }\end{array}$ & $\begin{array}{l}\text { Dificultades de } \\
\text { participación y } \\
\text { movilización }\end{array}$ \\
\hline $\begin{array}{l}\text { Buenas prácticas } \\
\text { alimentarias }\end{array}$ & $\begin{array}{l}\text { Número de niños } \\
\text { que están a cargo } \\
\text { de cuidadoras (dife- } \\
\text { rentes a los padres) }\end{array}$ & $\begin{array}{l}\text { Estilos de vida } \\
\text { saludables }\end{array}$ & $\begin{array}{l}\text { Número de padres } \\
\text { que conocen los } \\
\text { programas orienta- } \\
\text { dos a niños de } 0 \text { a } \\
5 \text { años. }\end{array}$ & $\begin{array}{l}\text { Salud mental - } 5 \\
\text { trastornos de mayor } \\
\text { incidencia en niños } \\
\text { y madre gestante }\end{array}$ \\
\hline $\begin{array}{l}\text { Generar buenos } \\
\text { hábitos físicos, } \\
\text { psicológicos y } \\
\text { emocionales por } \\
\text { medio de activi- } \\
\text { dades lúdicas }\end{array}$ & Educación sexual & Salud preventiva. & $\begin{array}{l}\text { Porcentaje de } \\
\text { menores de } 6 \text { años } \\
\text { en educación inicial } \\
\text { pública y privada }\end{array}$ & $\begin{array}{l}\text { Vivienda digna: } \\
\text { servicios públicos, } \\
\text { espacios dignos y } \\
\text { estrato adecuado }\end{array}$ \\
\hline
\end{tabular}




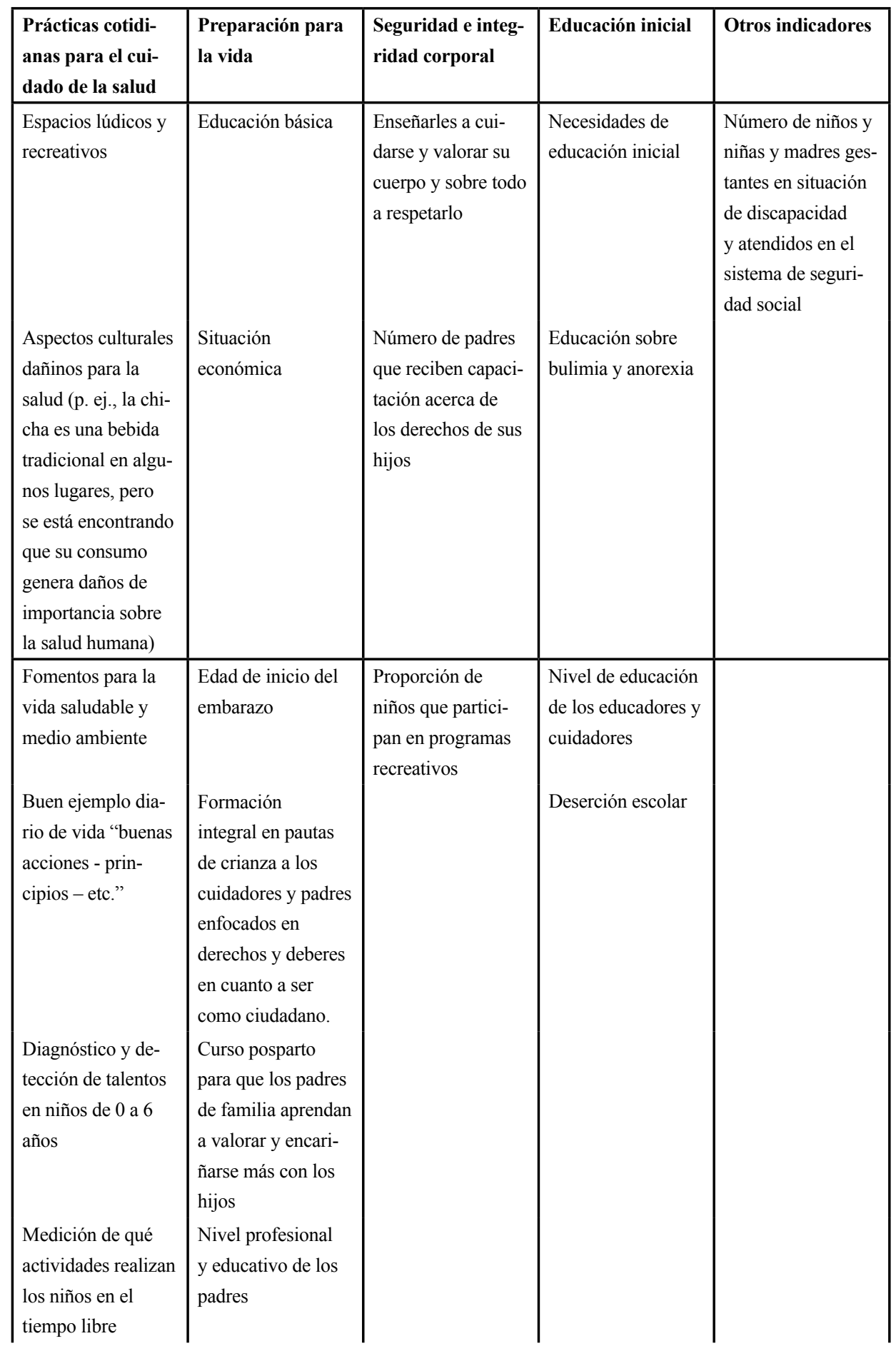




\begin{tabular}{|l|l|l|l|l|}
\hline $\begin{array}{l}\text { Prácticas cotidi- } \\
\text { anas para el cui- } \\
\text { dado de la salud }\end{array}$ & $\begin{array}{l}\text { Preparación para } \\
\text { la vida }\end{array}$ & $\begin{array}{l}\text { Seguridad e integ- } \\
\text { ridad corporal }\end{array}$ & Educación inicial & Otros indicadores \\
\hline $\begin{array}{l}\text { Número de redes } \\
\text { sociales de apoyo } \\
\text { que operan dirigi- } \\
\text { das a menores de } \\
6 \text { años } \\
\text { Número de con- } \\
\text { troles prenatales } \\
\text { Proporción de ni- } \\
\text { nos con educación } \\
\text { inicial } \\
\text { Número de niños } \\
\text { que asisten a los } \\
\text { programas de salud }\end{array}$ & & & & \\
\hline
\end{tabular}

Fuente: elaboración propia con base en los resultados de la investigación 